\title{
AVALIAÇÃO DO TAMANHO DE BOLHA PRODUZIDO POR AERADORES TIPO TUBO POROSO E MICRO-VENTURI EM COLUNA DE FLOTAÇÃO MINIPILOTO
}

\author{
A. S. TROIAN ${ }^{1}$, C. WIEHE ${ }^{1}$, C. OLIVEIRA ${ }^{2}$, R. T. RODRIGUES ${ }^{1 *}$ \\ ${ }^{1}$ Universidade Federal do Rio Grande do Sul (UFRGS), Departamento de Engenharia de Minas \\ ${ }^{2}$ UniLaSalle, Mestrado em Avaliação em Impactos Ambientais em Mineração. \\ rafael.teixeira@ufrgs.br*
}

Artigo submetido em novembro/2015 e aceito em dezembro/2015

DOI: $10.15628 /$ holos.2015.3682

\section{RESUMO}

O tipo de aerador (sparger), assim como a dosagem de espumante, possuem um papel fundamental no controle do tamanho de bolha em colunas de flotação, com influências na cinética de flotação e no desempenho metalúrgico (recuperação e teor). Este trabalho apresenta estudos de avaliação online da distribuição de tamanho de bolhas geradas por um sparger tipo tubo poroso e um micro-venturi acoplados em uma coluna de flotação minipiloto automatizada. Os efeitos da dosagem de espumante DF250 (éter metílico de polipropilenoglicol) e da vazão de ar no tamanho de bolha e no holdup foram avaliados. $O$ diâmetro médio de bolha foi fortemente influenciado pela dosagem de espumante para ambos os spargers; entretanto o micro-venturi foi mais eficiente do que o tubo poroso, por dispersar o ar mais finamente e com menores dosagens de espumante. Os menores diâmetros médios obtidos, de acordo com as condições experimentais aplicadas, foram de $1,25 \mathrm{~mm}$ $(154,8 \mathrm{mg} / \mathrm{L}$ de DF250) para o tubo poroso e $0,45 \mathrm{~mm}$ $(44,2 \mathrm{mg} / \mathrm{L}$ de DF250) para o micro-venturi.

PALAVRAS-CHAVE: flotação em coluna, parâmetros operacionais, análise de imagens, controle e automação.

\section{EVALUATION OF THE BUBBLE SIZE PRODUCED BY A POROUS TUBE AND A MICRO- VENTURI IN A MINI PILOT COLUMN FLOTATION}

\begin{abstract}
The sparger type as well as the frother dosage have a fundamental role on the bubble size control with influences on the kinetic flotation and metallurgical performance (recovery and grade). This work shows online evaluation studies of the the bubble size distribution generated by a porous tube sparger and a micro-venturi coupled in an automatized mini pilot flotation column. The effects of the frother (DF250 propylene glycol methyl ether) dosage and the air flow rate on the bubble size and holdup were evaluated. The
\end{abstract}

mean bubble diameter was strongly influenced by the frother dosage for both spargers; nevertheless, the micro-venturi was more efficient than the porous tube, because it was able to disperse the air more finely and with smaller dosages of frother. The smaller mean diameters obtained, according to the applied experimental conditions, were of $1.25 \mathrm{~mm}(154.8 \mathrm{mg} / \mathrm{L}$ of DF250) for the porous tube and $0.45 \mathrm{~mm}(44.2 \mathrm{mg} / \mathrm{L}$ of DF250) for the micro-venturi.

KEYWORDS: column flotation, operating parameters, image analysis, automation and control. 


\section{INTRODUÇÃO}

Os parâmetros relacionados à dispersão ar-polpa no processo de flotação incluem, principalmente, medidas de velocidade superficial de gás $(\mathrm{Jg})$, do tamanho de bolhas (normalmente o diâmetro médio de Sauter, $D_{32}$ ), do holdup de gás $(\varepsilon g)$ e da estimativa do fluxo superficial de bolhas (Sb) (Finch et al., 2000), definido como:

$$
\mathrm{Sb}=\frac{6 \cdot \mathrm{Jg}}{\mathrm{D}_{32}}
$$

Dentre as técnicas propostas para avaliação do tamanho de bolha, os métodos que utilizam o conceito da visualização de bolhas em uma câmara externa, combinando técnicas de captura, processamento e análise de imagens digitais aparecem como soluções promissoras para medições online de tamanho de bolhas em células de flotação industriais (Chen et al., 2001; Grau e Heiskanen, 2002 e 2003; Hernandez-Aguilar et al., 2004, Rodrigues e Rubio, 2003; Rodrigues; 2004; Oliveira et al., 2011; Pompeo et al, 2013).

Os espumantes desempenham um papel fundamental no controle do tamanho de bolha e na estabilização da espuma (Cho e Laskowski, 2002). O tipo de sparger e as condições operacionais também influenciam fortemente a dispersão do ar (Oliveira et al., 2011; Pompeo et al, 2013). As colunas de flotação em escala de bancada e minipiloto utilizam, frequentemente, elementos porosos como sistema de geração de bolhas. Industrialmente, os principais spargers utilizados são do tipo injeção de misturas ar-água ou ar-polpa (recirculação) em constrições de fluxo (como por exemplo os sistemas de aeração USBM/Cominco, MinnovEX, Microcel ${ }^{T M}$ e o cavitation tube - CPT) (Finch, 1995). Este trabalho apresenta estudos para avaliação da distribuição de tamanho de bolha produzido por dois tipos de spargers (tubo poroso e micro-venturi), utilizando um sistema online de medição de tamanho de bolha em uma coluna de flotação minipiloto.

\section{METOdOLOGIA}

O tamanho de bolha foi determinado online e de modo dinâmico, ou seja, durante o ensaio a dosagem de tensoativo foi modificada para valores pré-determinados (escalonado) e monitorouse o tamanho de bolha produzido. O sistema experimental utilizado para determinação do tamanho de bolha foi o mesmo descrito nos trabalhos de Oliveira et al. (2011) e Pompeo et al. (2013). A câmara visualizadora de bolhas, confeccionada em alumínio, com dimensões $27 \times 16,5 \times 11 \mathrm{~cm}$ (altura x largura x profundidade) foi conectada à base de uma coluna de flotação minipiloto de 27,5 $\mathrm{mm}$ de diâmetro interno por $210 \mathrm{~mm}$ de altura (Fig. 1-A). As imagens foram capturadas com uma câmera SONY XC-HR70, acoplada a uma lente de zoom. As etapas de captura, processamento e análise das imagens foram automatizadas empregando um software de análise de imagens (Image Tool 3.0). O tempo para aquisição, processamento e análise de cada imagem foi de $10 \mathrm{~s}$. A iluminação do sistema foi realizada por uma placa de LED. 
A geração de bolhas foi realizada em uma câmara conectada na parte inferior da câmara de visualização de bolhas. A Figura 1 apresenta um esquema desta câmara com os spargers utilizados, tubo poroso (B) e micro-venturi (C). O tubo poroso, fabricado com pó de aço inox sinterizado, possuía medidas externas de $15 \times 30 \mathrm{~mm}$ (diâmetro $\times$ comprimento) e espessura de parede de 2,3 $\mathrm{mm}$. A vazão da solução de tensoativo injetado abaixo do tubo poroso foi de $300 \mathrm{~mL} / \mathrm{min}$. 0 microventuri possuía diâmetro de orifício igual a $0,8 \mathrm{~mm}$ e foi confeccionado em poliuretano. A vazão de água injetada no micro-venturi foi de $200 \mathrm{~mL} / \mathrm{min}$ e a pressão resultante foi de $147 \mathrm{kPa}$. Antes de entrar em operação todo o sistema foi preenchido com água. Todos os experimentos foram realizados a temperatura ambiente $\left(\sim 25^{\circ} \mathrm{C}\right)$, com água da rede municipal de abastecimento de Porto Alegre/RS (DMAE) e pH natural ( 6,5 - 7). O holdup foi determinado através de medidas de pressão realizadas por dois sensores de pressão instalados na zona de coleta da coluna de flotação, distantes $925 \mathrm{~mm}$ e $1970 \mathrm{~mm}$ do topo da coluna. Foram avaliados os efeitos da concentração de tensoativo DF250 (Éter Metílico de Polipropilenoglicol - $\mathrm{CH}_{3}\left(\mathrm{OC}_{3} \mathrm{H}_{6}\right)_{4} \mathrm{OH}$ ) e da vazão de ar no tamanho de bolha e no holdup.

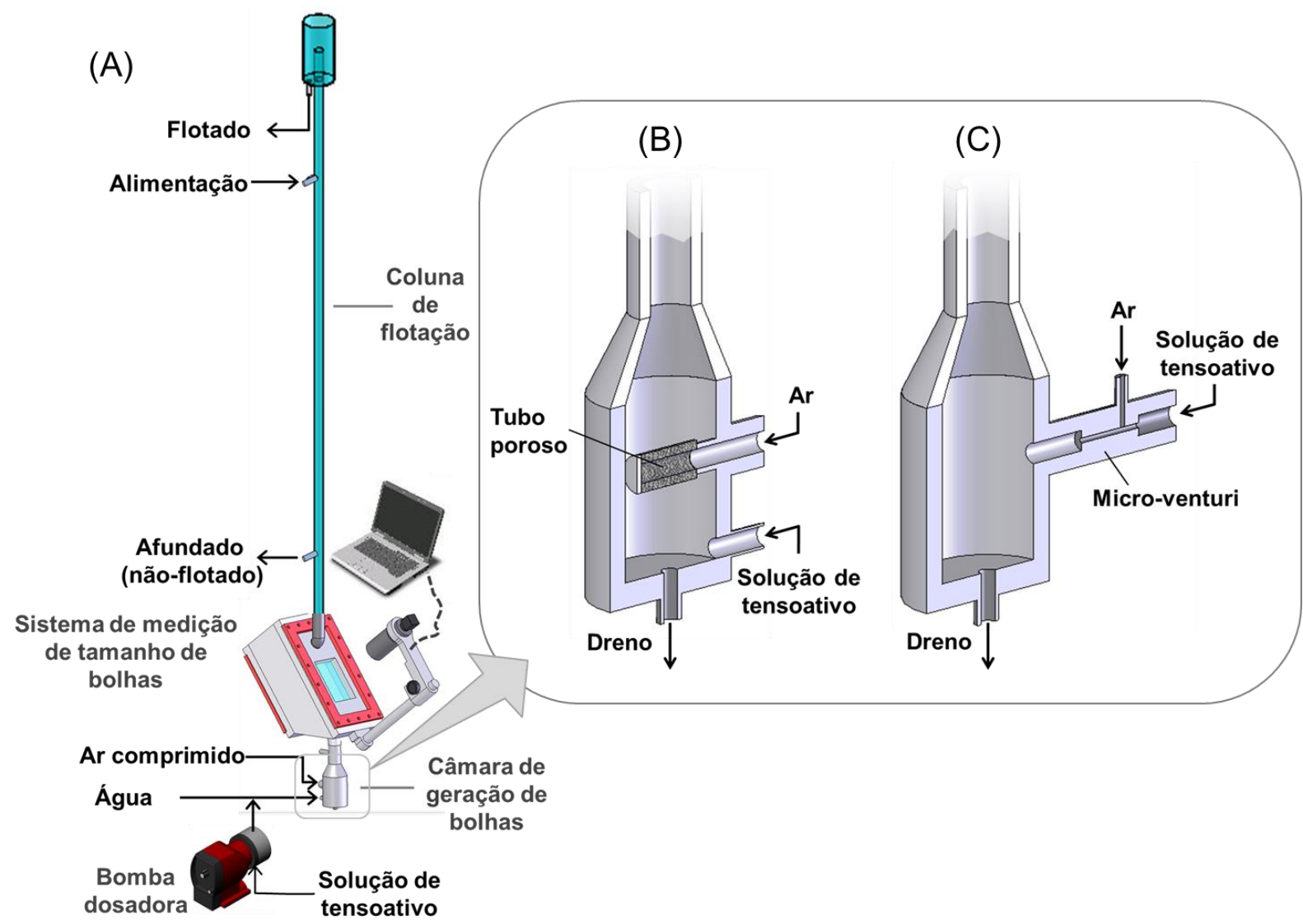

Figura 1. (A) Montagem experimental para determinação do tamanho de bolha. (B) Câmara de geração de bolhas com sparger tipo tubo poroso. (C) Câmara de geração de bolhas com sparger tipo micro-venturi. 
$\mathrm{Na}$ análise das imagens foi adotado o diâmetro de Feret médio (medido em 8 ângulos) como sendo o diâmetro de bolha. $O$ diâmetro médio adotado foi o diâmetro médio de Sauter $\left(D_{32}\right)$, definido como o diâmetro médio volume-superfície, conforme a Equação 1.

$$
\mathrm{d}_{\text {sauter }}=\frac{\Sigma \mathrm{n}_{\mathrm{i}} \mathrm{d}_{\text {bi }}^{3}}{\Sigma \mathrm{n}_{\mathrm{i}} \mathrm{d}_{\mathrm{bi}}^{2}}
$$

onde, os parâmetros $d_{b}$ e $n$ são o diâmetro de bolha e o número de bolhas para cada classe de tamanho " $i$ ", respectivamente.

\section{RESULTADOS E DISCUSSÕES}

As medidas de tamanho de bolha apresentaram um comportamento similar tanto para o tubo poroso quanto para o micro-venturi. A Figura 2 apresenta a variação do tamanho de bolha para diferentes dosagens do espumante DF250 para o sparger tipo tubo poroso. Os incrementos na dosagem de espumante de 0 a 154,8 mg/L resultaram numa redução gradual no diâmetro de bolha, com patamares definidos, desde $3,45 \mathrm{~mm}$ até $1,25 \mathrm{~mm}$. Observou-se também que o aumento da vazão de $\operatorname{ar}\left(Q_{\text {ar }}\right)$ de $120 \mathrm{ml} / \mathrm{min}(\mathrm{Jg}=0,35 \mathrm{~cm} / \mathrm{s})$ para $180 \mathrm{ml} / \mathrm{min}(\mathrm{Jg}=0,52 \mathrm{~cm} / \mathrm{s})$ não teve reflexo no tamanho de bolha; entretanto, observou-se um aumento no holdup (Figura 3), conforme previsto segundo o regime de borbulhamento uniforme (bubbly flow regime).

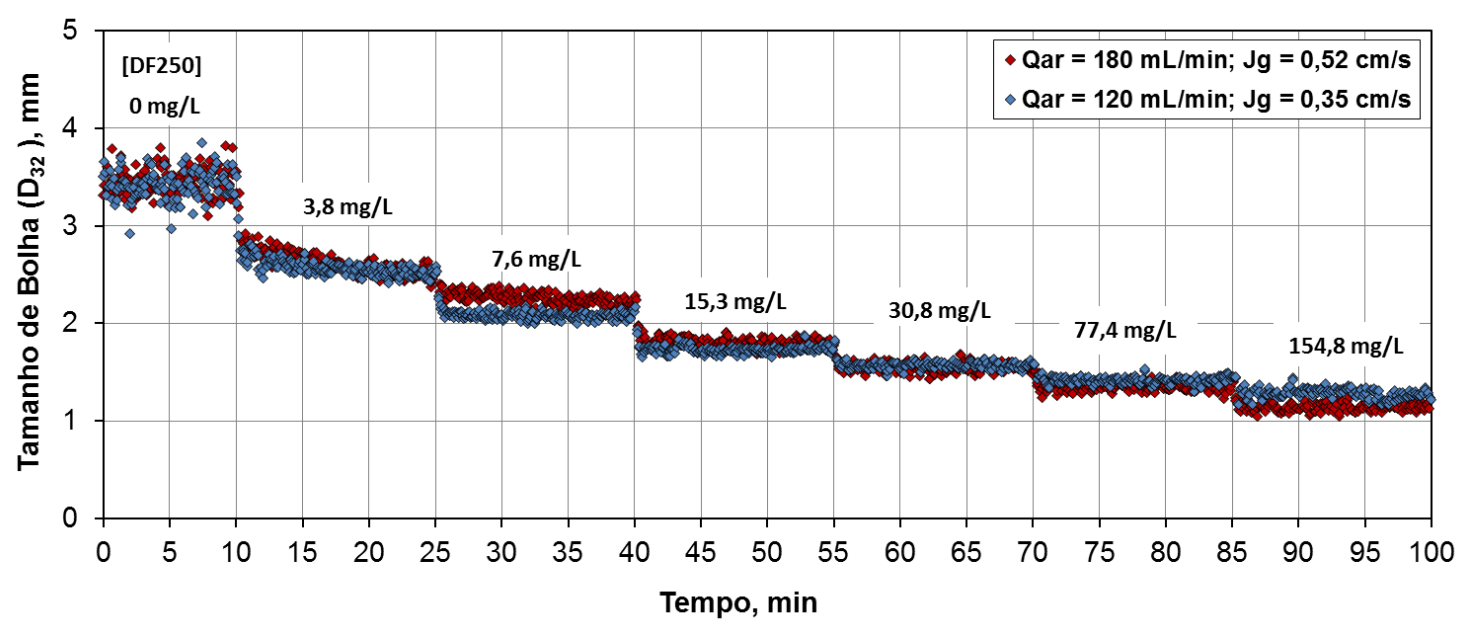

Figura 2. Variação do tamanho de bolha para diferentes dosagens de DF250 com sparger do tipo tubo poroso. 


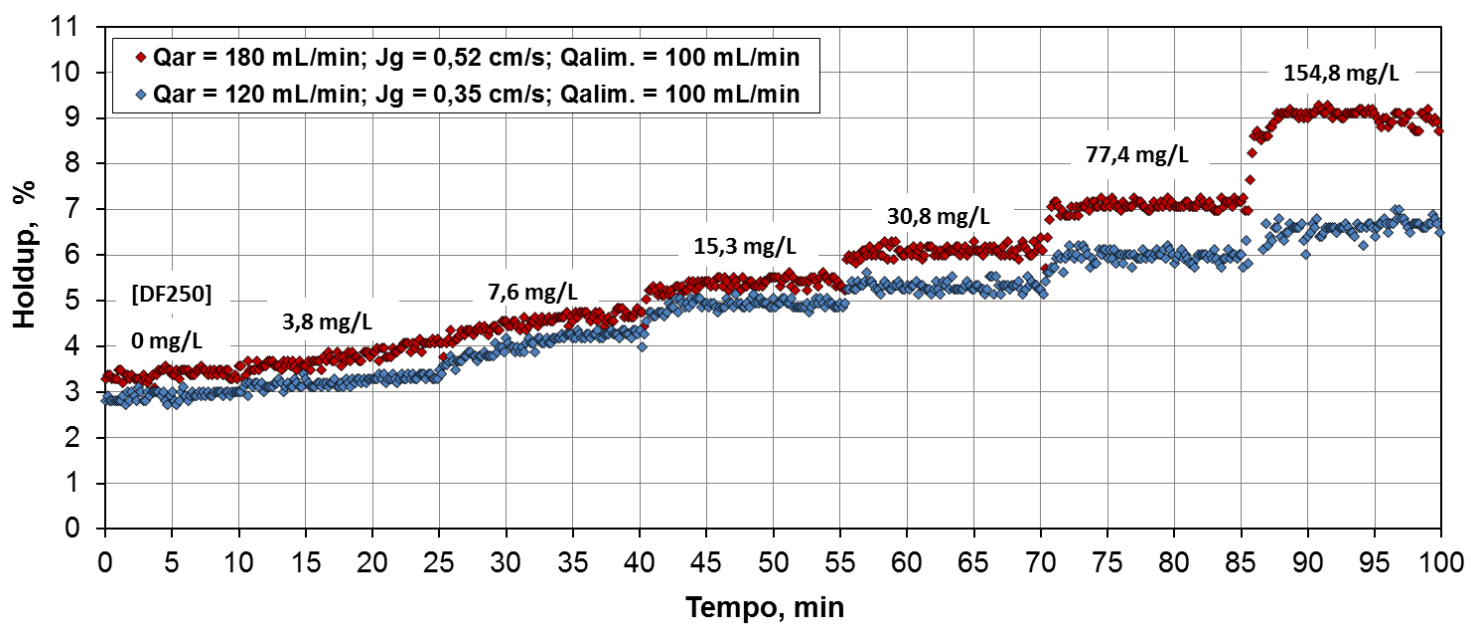

Figura 3. Variação do holdup para diferentes dosagens de DF250 com sparger do tipo tubo poroso.

A Figura 4 apresenta o histórico do tamanho de bolha para diferentes dosagens do espumante DF250 para o micro-venturi. O micro-venturi gerou bolhas desde $2,72 \mathrm{~mm}$ até $0,45 \mathrm{~mm}$ para dosagens de espumante de 0 até $44,2 \mathrm{mg} / \mathrm{L}$. Observou-se também que o aumento da vazão de $\operatorname{ar}\left(Q_{\text {ar }}\right)$ de $120 \mathrm{ml} / \mathrm{min}(\mathrm{Jg}=0,35 \mathrm{~cm} / \mathrm{s})$ para $180 \mathrm{ml} / \mathrm{min}(\mathrm{Jg}=0,52 \mathrm{~cm} / \mathrm{s})$ não teve reflexo no tamanho de bolha para concentrações a partir de $14,7 \mathrm{mg} / \mathrm{L}$, entretanto, observou-se um aumento no holdup, como ilustrado na Figura 5, devido ao aumento na vazão de ar.

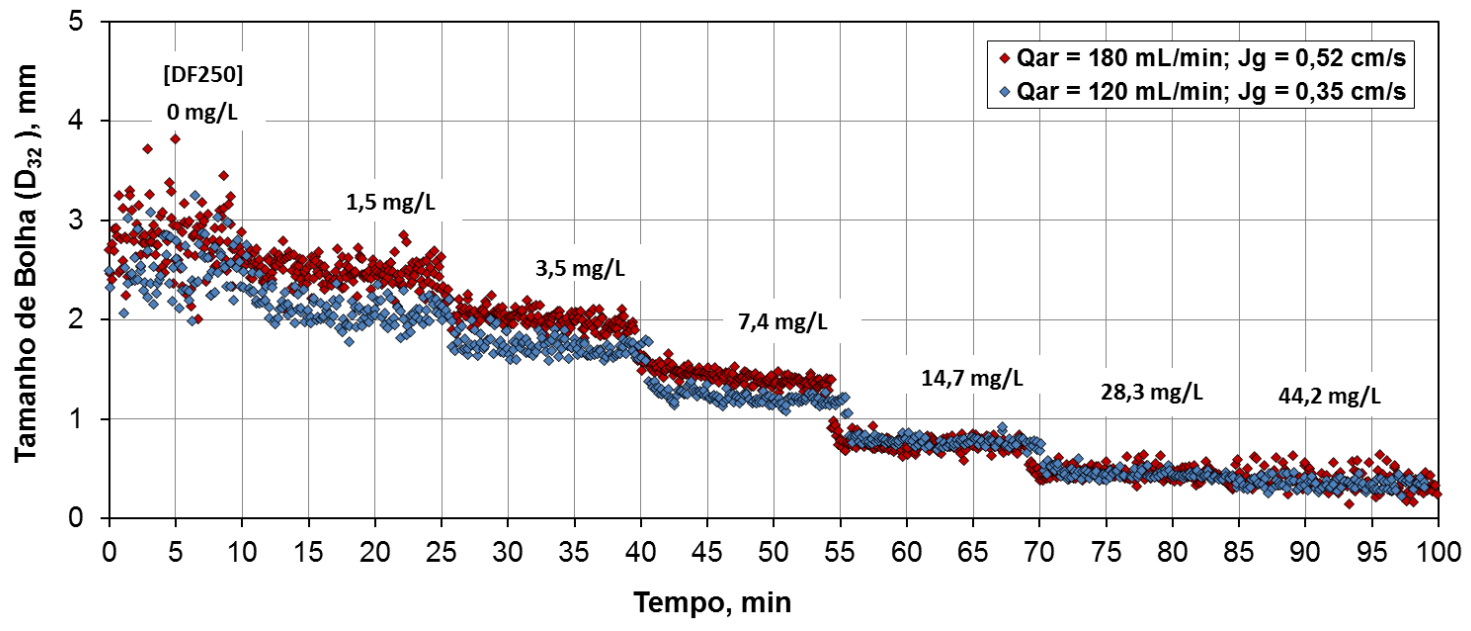

Figura 4. Variação do tamanho de bolha para diferentes dosagens de DF250 com sparger do tipo micro-venturi.

A Figura 6 mostra claramente que o micro-venturi é capaz de gerar bolhas menores do que o tubo poroso com menores dosagens de espumante. Observa-se nas Figuras 8 e 9 que a distribuição de tamanho de bolha para dosagens similares de DF250 (aproximadamente $30 \mathrm{mg} / \mathrm{L}$ ), o microventuri foi capaz de produzir em torno de $60 \%$ das bolhas (base volume) menores do que $0,6 \mathrm{~mm}$, ao passo que para o tubo poroso a produção de bolhas nesta faixa de tamanho foi desprezível. As 
Figuras 10 e 11 também mostram que para a produção de bolhas da ordem de 1,4 mm, a dosagem de DF250 foi de 7,4 e 77,4 mg/L para o micro-venturi e tubo poroso, respectivamente.

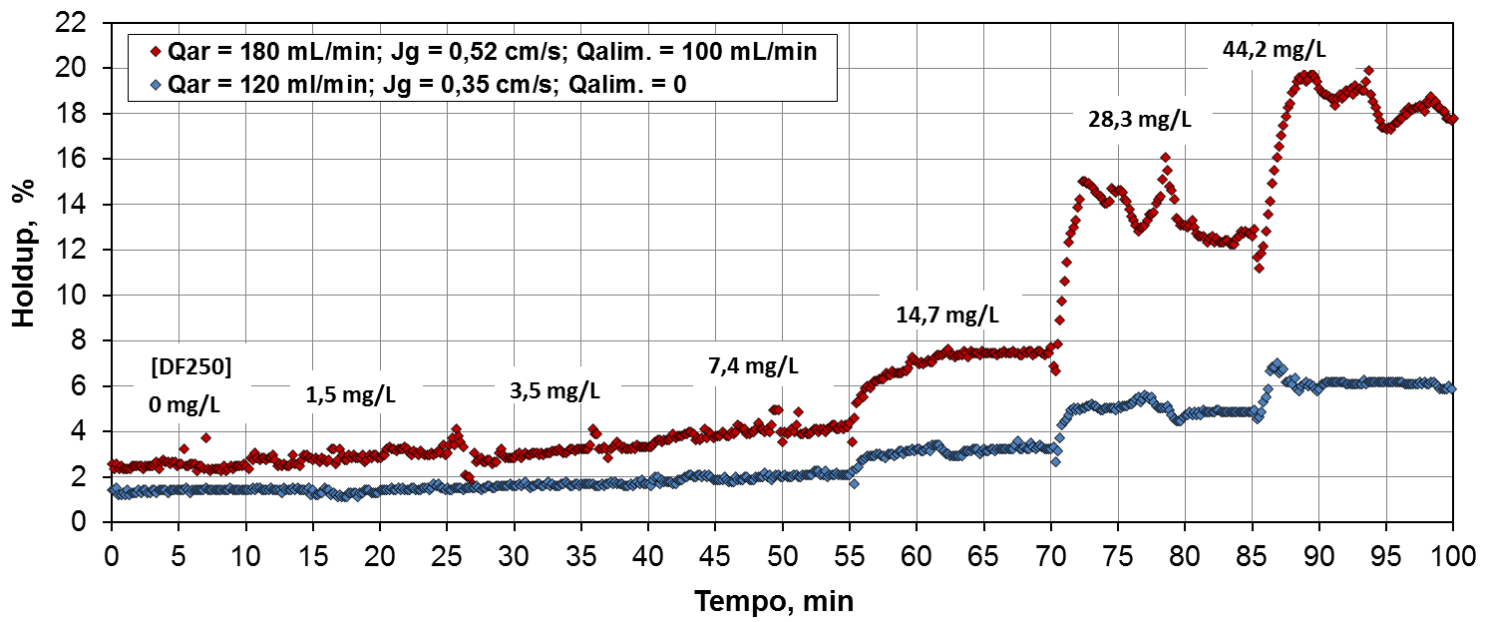

Figura 5. Variação do holdup para diferentes dosagens de DF250, com sparger do tipo micro-venturi.

Embora o venturi seja capaz de gerar bolhas mais finas, não foram observados valores maiores de holdup (Figura 7); isto deve-se a maior coalescência das bolhas na câmara de visualização e na coluna de flotação, pois o mesmo utiliza menores dosagens de espumante para obter diâmetros médios de bolha equivalente aos fornecidos pelo tubo poroso. Este inconveniente pode ser minimizado se a concentração de tensoativo na câmara de visualização de bolhas estiver acima da concentração crítica de coalescência (CCC), conforme reportado por Cho e Laskowski (2002).

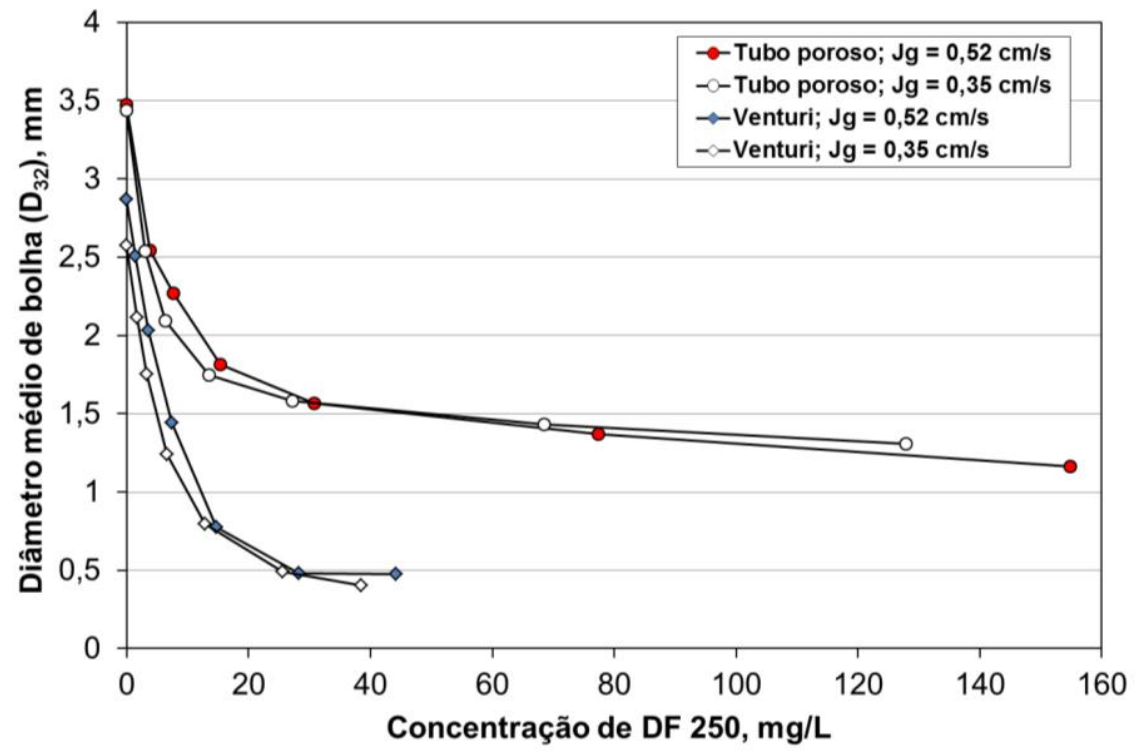

Figura 6. Efeito da dosagem de DF 250 no diâmetro médio de bolha. 


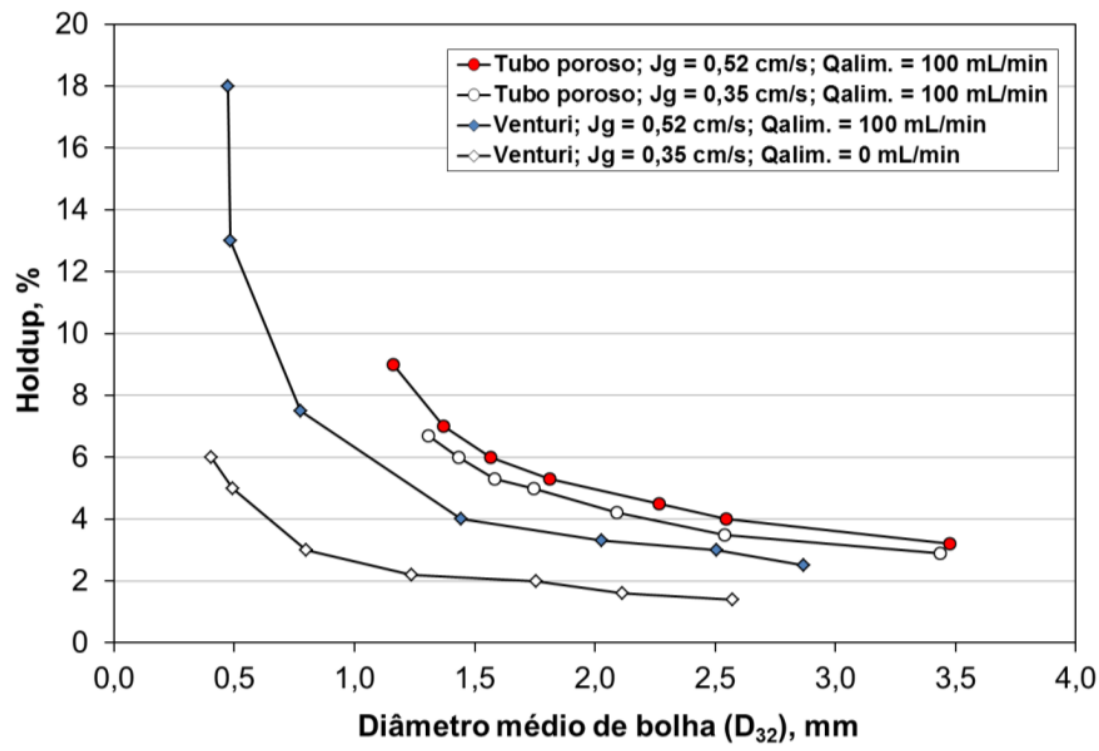

Figura 7. Efeito do diâmetro médio de bolha no holdup.
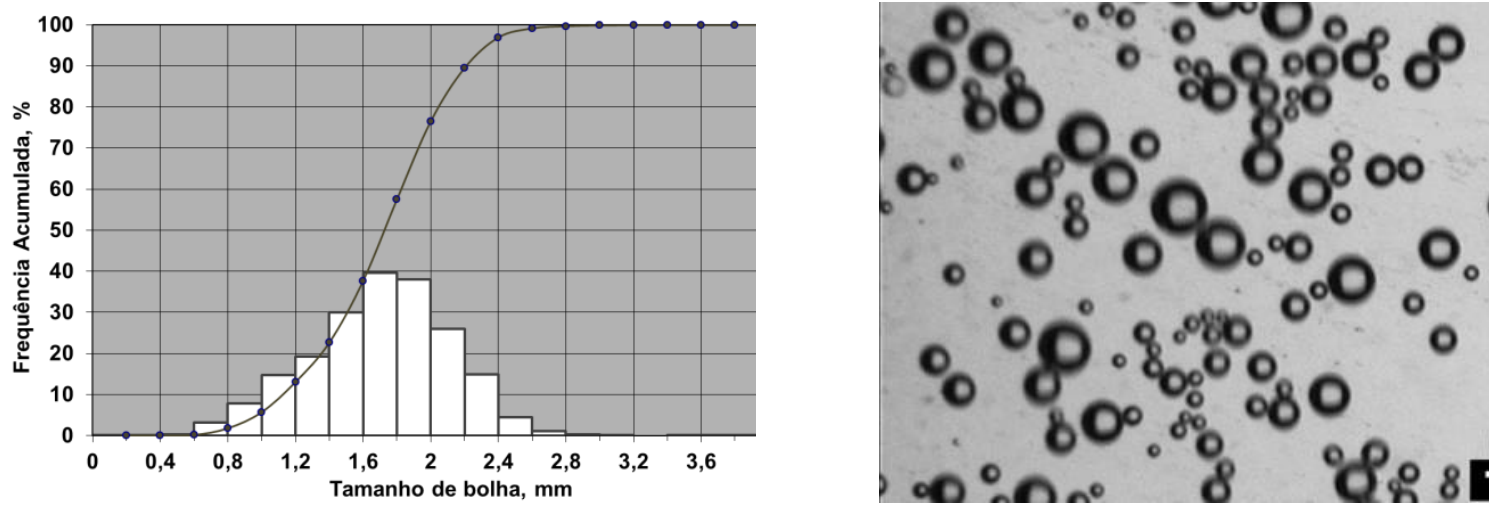

Figura 8. Distribuição de tamanho de bolha e típica imagem capturada. Condições experimentais: Tubo poroso, $[D F 250]=30,8 \mathrm{mg} / \mathrm{L} ; D_{32}=1,58 \mathrm{~mm} ; \mathrm{Jg}=0,52 \mathrm{~cm} / \mathrm{s}$.
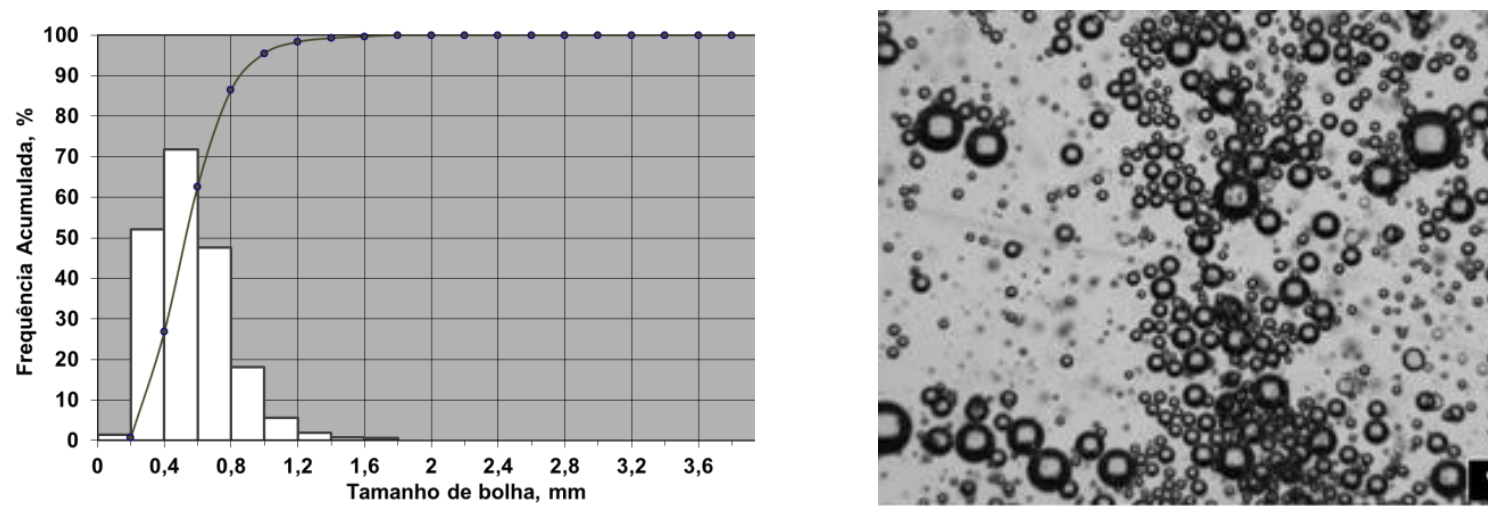

Figura 9. Distribuição de tamanho de bolha e típica imagem capturada. Condições experimentais: Microventuri, $[D F 250]=28,3 \mathrm{mg} / \mathrm{L} ; D_{32}=0,49 \mathrm{~mm} ; \mathrm{Jg}=0,52 \mathrm{~cm} / \mathrm{s}$. 

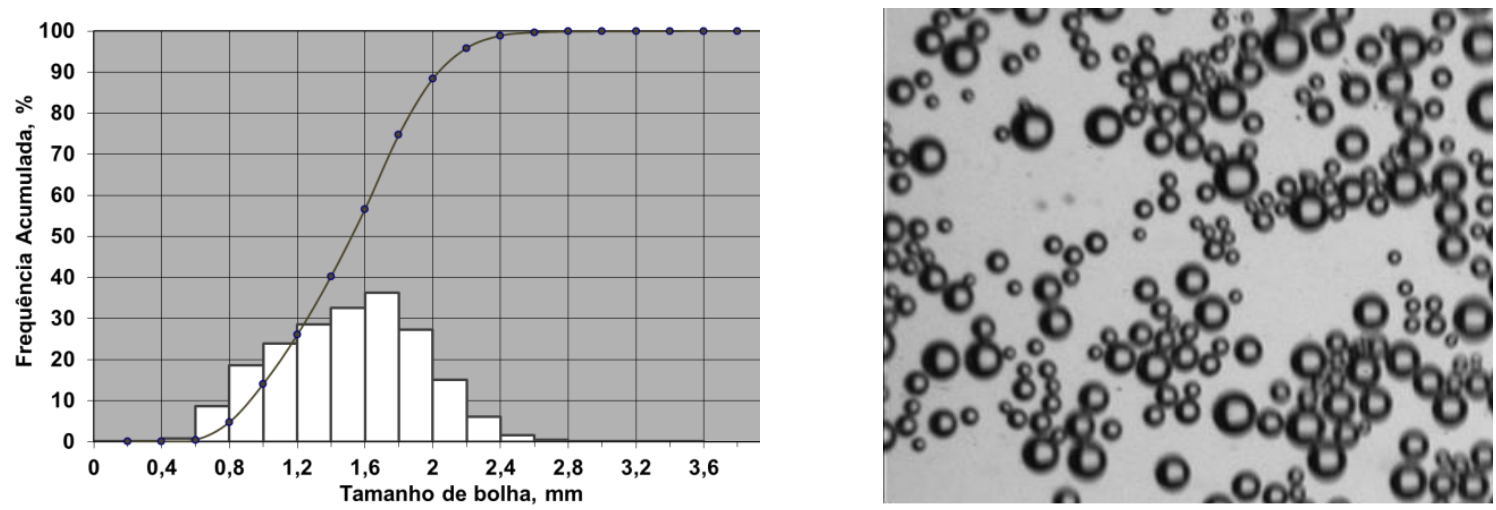

Figura 10. Distribuição de tamanho de bolha e típica imagem capturada. Condições experimentais: Tubo poroso, $[D F 250]=77,4 \mathrm{mg} / \mathrm{L} ; D_{32}=1,37 \mathrm{~mm} ; \mathrm{Jg}=0,52 \mathrm{~cm} / \mathrm{s}$.
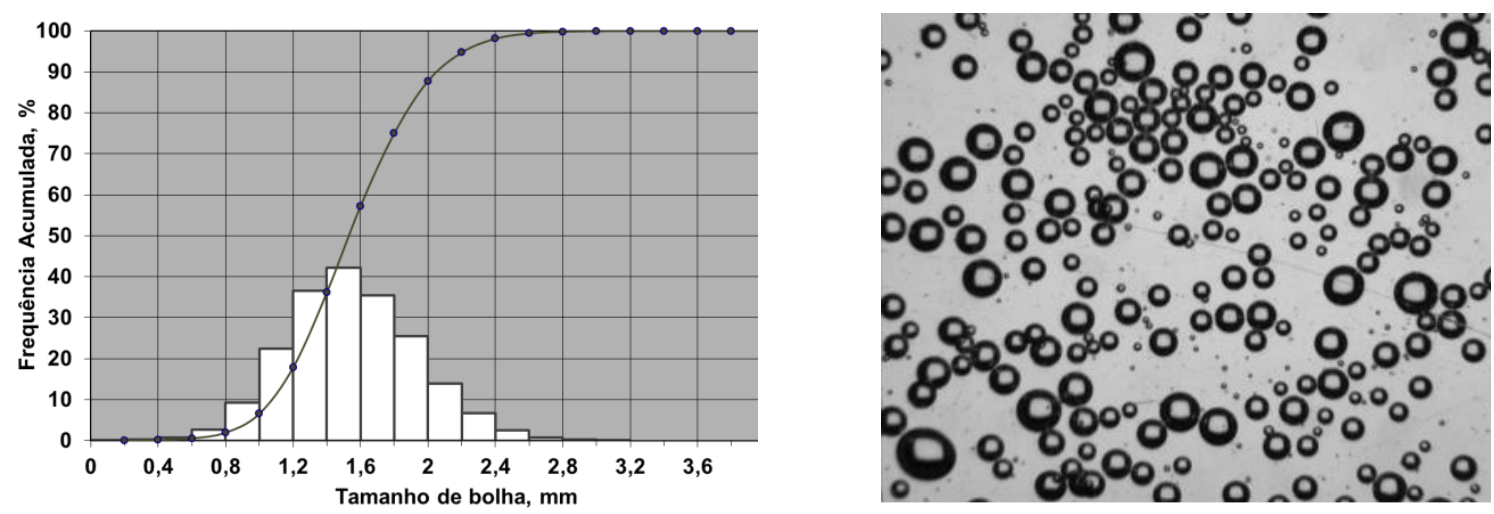

Figura 11. Distribuição de tamanho de bolha e típica imagem capturada. Condições experimentais: Micro-venturi, $[D F 250]=7,4 \mathrm{mg} / \mathrm{L} ; D_{32}=1,44 \mathrm{~mm} ; \mathrm{Jg}=0,52 \mathrm{~cm} / \mathrm{s}$.

\section{CONCLUSÕES}

O diâmetro médio de bolha foi fortemente influenciado pela dosagem de espumante para ambos os spargers; entretanto o micro-venturi foi mais eficiente do que o tubo poroso, pois dispersa o ar mais finamente e com menores dosagens de espumante.

As medidas de holdup indicaram que devem ser mantidas concentrações de espumante superiores à concentração crítica de coalescência (CCC) para reduzir a coalescência das bolhas após a sua geração.

\section{REFERÊNCIAS BIBLIOGRÁFICAS}

1. CHEN, F., GOMEZ, C.O., FINCH, J.A. Bubble size measurement in flotation machines. Minerals Engineering, v.14, n.4, p.427-432, 2001.

2. CHO, Y.S., LASKOWSKI, J.S. Effect of flotation frothers on bubble size and foam stability. International Journal of Mineral Processing, v.64, p.69-80, 2002. 
3. FINCH, J.A., Column flotation: A selected review - Part IV: Novel flotation devices. Minerals Engineering, v.8, n.6, p.587-602, 1995.

4. FINCH, J. A., DOBBY, G. S., "Column Flotation", Pergamon Press, 1990, p. 180.

5. FINCH, J., XIAO, J., HARDIE, C., GOMEZ, C.O. Gas dispersion properties: Bubble surface area flux and gas holdup. Minerals Engineering, v.13, n.4, p.365-372, 2000.

6. GRAU, R.A., HEISKANEN, K. Visual technique for measuring bubble size in flotation machines. Minerals Engineering, v.15, p.507-513, 2002.

7. GRAU, R.A., HEISKANEN, K. Gas dispersion measurements in a flotation cell. Minerals Engineering, v.16, p.1081-1089, 2003.

8. HERNANDEZ-AGUILAR, J.R., COLEMAN, R.G., GOMEZ, C.O., FINCH, J.A. A comparison between capillary and imaging techniques for sizing bubbles in flotation systems. Minerals Engineering, v.17, n.1, p.53-61, 2004.

9. OLIVEIRA, C., SEGER, M.A., RODRIGUES, R.T. Desenvolvimento de um equipamento para avaliação da dispersão de bolhas em células de flotação. In: Encontro Nacional de Tratamento de Minérios e Metalurgia Extrativa. Anais do XXIV Encontro Nacional de Tratamento de Minérios e Metalurgia Extrativa; 2011; Salvador, Brasil. p. 115-124.

10. POMPEO, A.A., OLIVEIRA, C., SEGER, M.A., LOTTERMANN, M.R., RODRIGUES, R.T. Avaliação do tamanho de bolha produzido por um sparger cominco. In: Encontro Nacional de Tratamento de Minérios e Metalurgia Extrativa. Anais do XXV Encontro Nacional de Tratamento de Minérios e Metalurgia Extrativa; 2013; out. 20-24: Goiânia, Brasil. p. 61-69.

11. RODRIGUES, R.T., RUBIO, J. New basis for measuring the size distribution of bubbles. Minerals Engineering 2003; 16;757-765.

12. RODRIGUES, R.T. Desenvolvimento da Técnica LTM-BSizer para a Caracterização de Bolhas e Avaliação de Parâmetros no Processo de Flotação. [Tese de Doutorado]. Programa de PósGraduação em Engenharia de Minas, Metalúrgica e de Materiais - PPGEM/UFRGS; 2004. 